\title{
123 Autismo: Um aplicativo móvel para auxiliar no ensino de habilidades iniciais da matemática a crianças com autismo
}

\author{
Lukas Teixeira Carvalho', Mônica Ximenes Carneiro da Cunha' \\ ${ }^{1}$ Instituto Federal de Educação, Ciência e Tecnologia de Alagoas (IFAL) \\ Campus Maceió - Maceió - AL - Brasil \\ \{bsi.lukas, mxccunha\}@gmail.com
}

\begin{abstract}
Technological applications uses has been provided great transformations and numerous benefits, both in the educational area as in the treatment of several syndromes, among them, autism. Thus, the 123 Autism application, aimed at teaching math skills for children with autism, was elaborated based on the therapeutic programs premises with scientific evidence, such as: Behavior Analysis and Structured Teaching. The application was developed using Corona SDK framework, with specially designed interfaces using Adobe Illustrator and Corel Draw. The app final version is available for download from the Google Play Store.
\end{abstract}

Resumo. O uso de aplicativos tecnológicos tem proporcionado grandes transformações e inúmeros benefícios, tanto na área educacional quanto no tratamento de diversas síndromes, entre elas o autismo. Assim, o aplicativo 123 Autismo, voltado ao ensino de habilidades matemáticas para crianças com autismo, foi elaborado com base nas premissas dos programas terapêuticos com evidências científicas, tais como: Análise Comportamental e Ensino Estruturado. O aplicativo foi desenvolvido usando a estrutura Corona SDK, com interfaces especialmente projetadas usando o Adobe Illustrator e o Corel Draw. A versão final do aplicativo está disponivel para download na Google Play Store.

\section{Cenário de uso}

O Transtorno do Espectro do Autismo (TEA) é caracterizado como um transtorno invasivo do desenvolvimento que se manifesta na primeira infância e perdura por toda a vida, englobando déficits qualitativos na comunicação e interação social. Além disso, o indivíduo com autismo apresenta padrões de comportamento repetitivos $\mathrm{e}$ estereotipados e um repertório restrito de atividades (SCHWARTZMAN, 2011).

O autismo consiste em um transtorno pervasivo e permanente, para o qual ainda não existe cura, ainda que a intervenção precoce possa alterar o prognóstico e suavizar os sintomas (SOCIEDADE BRASILEIRA DE PEDIATRIA, 2019). O diagnóstico do autismo é obtido através de observação com base nos critérios estabelecidos pela 5a edição do Manual de Diagnóstico e Estatística das Perturbações Mentais (DSM-V): a) déficits persistentes na comunicação social e na interação social em múltiplos contextos; b) padrões restritos e repetitivos de comportamento, interesses ou atividades; c) sintomas devem estar presentes precocemente no período do desenvolvimento; d) sintomas causam prejuízo clinicamente significativo no funcionamento social, profissional ou em outras áreas importantes da vida do indivíduo (APA, 2014, p. 50). 
Os comprometimentos advindos do autismo impactam no processo de ensinoaprendizagem que utiliza métodos convencionais. Pessoas com TEA se distraem ou se desconcentram facilmente (FERREIRA; FRANÇA, 2017) e têm dificuldade com foco atencioso, concentração e raciocínio lógico que são essenciais para aprendizagem de conceitos matemáticos (REDERD; SANTOS; HEES, 2018). Por isso, o ensino voltado a pessoas com autismo exige uma adaptação na aplicação das atividades e nas ações com o objetivo de promover um aprendizado eficaz.

Muitos indivíduos com TEA têm deficiências cognitivas significativas, embora alguns apresentem Quociente de Inteligência (QI) acima da média. As habilidades de aprendizagem, pensamento e resolução de problemas das pessoas com TEA podem variar de superdotadas a severamente comprometidas; desta forma, o tratamento deve ser individualizado e baseado em uma compreensão personalizada para cada indivíduo (KWEE, 2006). O tratamento multidisciplinar alicerçado em metodologias amplamente testadas e mundialmente utilizadas promove resultados bastante animadores, favorecendo uma compreensão de mundo a essas crianças, que muito colabora com a sua alfabetização (ORRÚ, 2009). Segundo Mello et al. (2013), as metodologias que mais se destacam e são mais utilizadas dentro das instituições de apoio ao autismo no Brasil são: TEACCH (Tratamento e Educação para Autistas e Crianças com Déficits relacionados com a Comunicação), que é baseado no Ensino Estruturado e envolve as esferas de atendimento educacional e clínico, ABA (Análise do Comportamento Aplicada) e PECS (Sistema de Comunicação por Troca de Figuras).

O aplicativo 123 Autismo adota, em suas telas de atividades, três mecanismos do Ensino Estruturado, ressaltados por Mesibov e Shea (2010): (a) estruturação do ambiente e das atividades de maneira que sejam compreensíveis para o indivíduo; (b) utilização dos pontos fortes relativos a habilidades visuais das pessoas com TEA; (c) observação dos interesses especiais dos indivíduos para envolvê-los na aprendizagem. Essas premissas servem como apoio para que o indivíduo com autismo possa superar os déficits característicos do TEA e visam atender as necessidades da educação especial, proporcionando uma melhor organização e estruturação da atividade, bem como a escolha de tarefas com grau de dificuldade adequado à capacidade da criança.

O ensino de matemática tem se mostrado desafiador para escola comum, com muitos alunos típicos apresentando dificuldades de aprendizado. Diversas iniciativas voltadas a aplicativos e jogos têm sido propostas nos últimos anos para tentar mitigar esse problema. O trabalho de Pires et al (2018), por exemplo, consiste em um jogo cujos personagens precisam desviar de obstáculos, solucionar problemas sobre as quatro operações da matemática e usar suas bolas especiais de tricô contra as criaturas mortasvivas. O trabalho de Beltrame et al (2018) propõe um jogo educacional para facilitar o aprendizado de matemática para alunos do ensino médio, ressaltando as dificuldades que enfrentam com o aprendizado desta matéria.

Foram realizadas buscas por aplicativos ou jogos voltados a auxiliar o processo ensinoaprendizagem de crianças com autismo e alinhados com as premissas do ensino estruturado. E, dentre os que sinalizaram em seus relatos aderência a base teórica, podese citar: ABC AUTISMO Clássico (FARIAS; SILVA; CUNHA, 2014), ABC Fônico (GARCIA; WELFER, 2018) e o Jogo ACA (GOBBO et al, 2018).

É neste contexto que foi desenvolvido o aplicativo 123 Autismo, para auxiliar no processo de ensino de habilidades básicas da matemática para pessoas com autismo, 
com diversos estilos de atividades visando trabalhar habilidades de transposição, correspondência, pareamento, identificação de número e quantidade, nomeação do número, sequência numérica e operações básicas de soma.

O aplicativo dispõe de uma interface intuitiva com elementos gráficos personalizados e atrativos, estruturados utilizando um padrão de esquerda (área de armazenamento) para direita (área de execução), com uso de cores como estratégia de esvanecimento de dicas, para poder captar toda a atenção do usuário e evitar que ele possa se distrair com outras funcionalidades do dispositivo móvel. Além disso, o aplicativo está dividido em quatro níveis de complexidade, com objetivos diferentes para estimular habilidades específicas dos indivíduos, a fim de propiciar a aquisição de conhecimentos e comportamentos, de uma forma adaptada às suas necessidades, que contribuem com o desenvolvimento cognitivo e a sua autonomia. Para reforçar e realizar a manutenção das habilidades adquiridas com a utilização do aplicativo, o usuário pode repetir a execução das atividades, mesmo se elas já estiverem concluídas.

\section{Desenvolvimento}

Primordialmente, foi necessário o entendimento das particularidades do universo autista, mediante revisões bibliográficas. O conhecimento dos sintomas, características, especificidades, peculiaridades e demais detalhes deste universo foi fundamental para que se pudesse propor funcionalidades no jogo, nas suas fases e níveis, para atender as reais necessidades do público alvo, que são crianças com autismo. Visando compreender de forma mais efetiva a problemática autista, foi realizada uma pesquisa de campo em uma associação de referência no atendimento a pessoas com autismo, localizada na Região Nordeste do Brasil, que adota o Ensino Estruturado como abordagem de tratamento e presta atendimento multidisciplinar a 45 crianças e adolescentes com TEA, cujo grau de comprometimento varia entre leve, moderado e severo.

Durante a pesquisa de campo, foram realizadas reuniões com integrantes da equipe multidisciplinar da instituição, composta por pedagogos, psicopedagogos e psicólogos, que ficaram em permanente contato com a equipe de desenvolvimento do aplicativo, devido às frequentes visitas in loco. Além das reuniões, foram realizados acompanhamentos dos atendimentos, com a devida autorização dos pais e da diretoria da associação. Durante as sessões de terapia, foi possível observar a aplicação prática das premissas do Ensino Estruturado, incluindo a elaboração das atividades, as técnicas utilizadas para evitar a distração da criança, o uso de itens de preferência de cada atendido para aumentar o interesse pela atividade, a hierarquia de dicas, a ordem das atividades, a complexidade dos níveis das tarefas utilizadas, quais os incentivos para a execução da atividade e recompensas após a sua execução.

Todas essas informações foram registradas, através de planilhas e uso de software de gestão de tarefas, como requisitos iniciais e fundamentais para tornar o jogo uma aplicação eficaz. Durante todas as etapas de desenvolvimento do 123 Autismo, foram realizados levantamentos, testes e validações com a equipe multidisciplinar e com o público-alvo a fim de garantir que as necessidades dos usuários fossem atendidas. Cabe ressaltar a importância das avaliações junto aos professores e terapeutas, uma vez que muitos dos atendidos na associação não puderam expressar as suas impressões verbalmente, devido a deficiência intelectual e a ausência de comunicação verbal, que 
VIII Congresso Brasileiro de Informática na Educação (CBIE 2019)

Anais dos Workshops do VIII Congresso Brasileiro de Informática na Educação (WCBIE 2019)

caracterizam um quadro de autismo severo. Após a identificação e especificação dos requisitos, foram elaborados os esboços das atividades, dos níveis e dos modelos das telas do jogo, apresentados na figura 1.

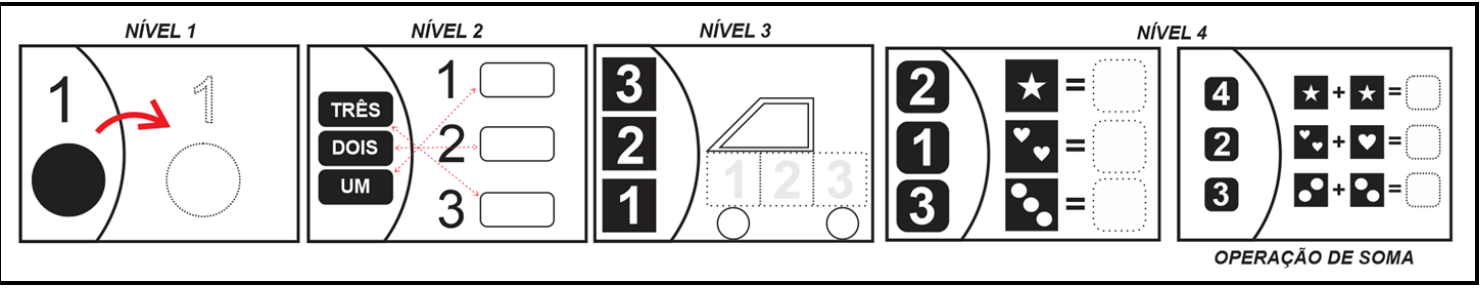

Figura 1. Esboços iniciais do aplicativo 123 Autismo.

A dinâmica da proposta para o jogo foi definida em: tela de abertura, tela para exibição dos níveis interligada ao menu de configuração de idiomas, tela das respectivas atividades de cada nível, tela de congratulações que é exibida a cada atividade concluída com êxito e serve como reforço positivo para estimular a continuidade da criança pelo jogo. Após a elaboração dos esboços, foram realizados os protótipos de alta fidelidade, conforme apresentado na figura 2 .

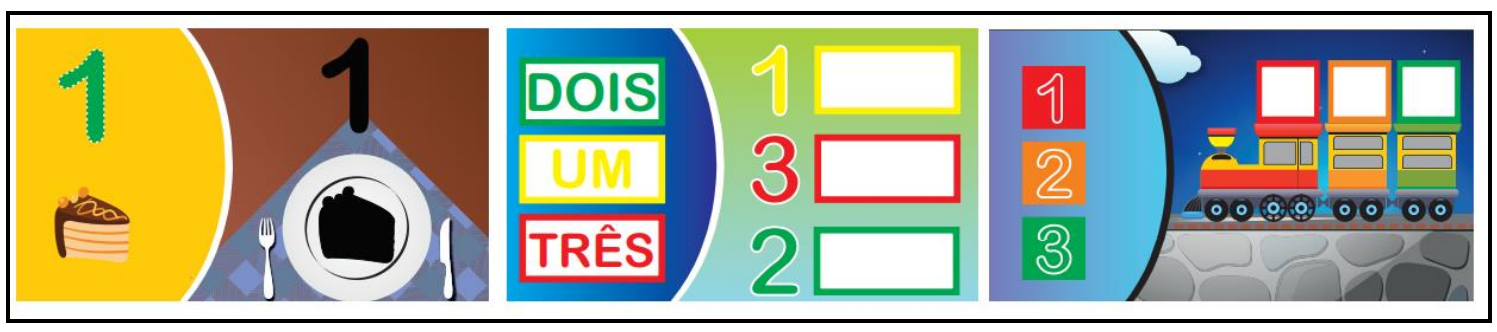

Figura 2. Protótipos de alta fidelidade do 123 Autismo.

Após a organização de todas as características do aplicativo, deu-se início a fase de desenvolvimento, onde foi utilizado o framework multiplataforma Corona SDK, escrito na linguagem LUA, para sua codificação. Este framework é capaz de compilar o mesmo código para diferentes plataformas, possibilitando assim disponibilizar o aplicativo para as plataformas Android, iOS (Sistema operacional da Apple), Desktops e Windows Phone (CORONA, 2019).

Tendo toda a base definida para o desenvolvimento do aplicativo, foram estabelecidos os períodos de implementação obedecendo à metodologia ágil Scrum, com gerenciamento através do aplicativo Trello e o WunderList. A etapa de desenvolvimento foi dividida em duas partes: a primeira fase com o intuito de produzir uma versão de testes para legitimar todas as funcionalidades do aplicativo com as características do ensino estruturado e realizar uma validação de usabilidade com crianças e a equipe multidisciplinar; a segunda fase foi destinada para implementar a versão final com os ajustes necessários após observação do uso por parte das crianças e também sugeridos por parte da equipe multidisciplinar.

\section{Apresentação do Software}

O aplicativo 123 Autismo é um aplicativo móvel, disponibilizado para Android e em fase de ajustes para $i O S$, cuja renda é revertida para a causa do autismo e manutenção da instituição parceira que colaborou com o desenvolvimento. $\mathrm{O}$ jogo busca ser o mais intuitivo possível e retrata de forma clara quais ações devem ser realizadas pelos 
VIII Congresso Brasileiro de Informática na Educação (CBIE 2019)

Anais dos Workshops do VIII Congresso Brasileiro de Informática na Educação (WCBIE 2019)

usuários durante a sua execução. Possui quatro níveis, com dez atividades cada, sequenciadas em ordem de complexidade crescente, medidas pela quantidade de acertos e estímulos exibidos a criança. Além de tudo, o aplicativo é de fácil configuração e está disponível em cinco idiomas: Português (de forma padrão), Inglês, Espanhol, Francês e Italiano, visando facilitar o acesso tanto em âmbito nacional quanto internacional. A equipe que participou da elaboração e desenvolvimento do 123 Autismo é a mesma do ABC Autismo Clássico, um aplicativo para ensino de pré-habilidades de leitura e escrita, que possui mais de 150 mil downloads no Brasil e no mundo.

As características do Ensino Estruturado são facilmente notadas na interface do aplicativo. O 123 Autismo, cuja tela inicial está apresentada na figura 3, possibilita uma fácil compreensão da proposta das atividades, estimula a autonomia, apresenta elementos consistentes e padrões fixos (áreas visualmente delimitadas e execução sempre na direção padrão da esquerda para a direita), potencializa o foco atencioso devido as telas atrativas e agradáveis e tem proposta alinhada ao modo convencional de atividades utilizadas no programa TEACCH, retratando o mais fidedignamente possível o ambiente utilizado pela criança durante a execução da cada atividade. As características anteriormente relatadas são de extrema importância visto que as pessoas com autismo não aceitam mudanças drásticas em suas rotinas e têm bastante dificuldade de concentração, necessitando, portanto, de todo um aparato para auxiliá-las.

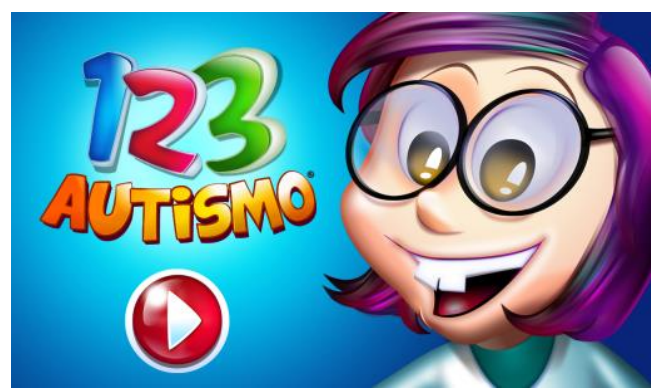

Figura 3. Tela de abertura do 123 Autismo.

O aplicativo possui 4 níveis, com 10 atividades sequenciadas em cada, com ordem crescente de complexidade e possibilita a coleta de até 30 estrelas que servem de estímulo para que o usuário permaneça engajado na utilização do jogo.

As telas evidenciadas na figura 4 estão relacionadas ao primeiro nível de atividades do aplicativo, que visa estimular a ação de transposição de elementos o número de vezes que representa a sua quantidade.

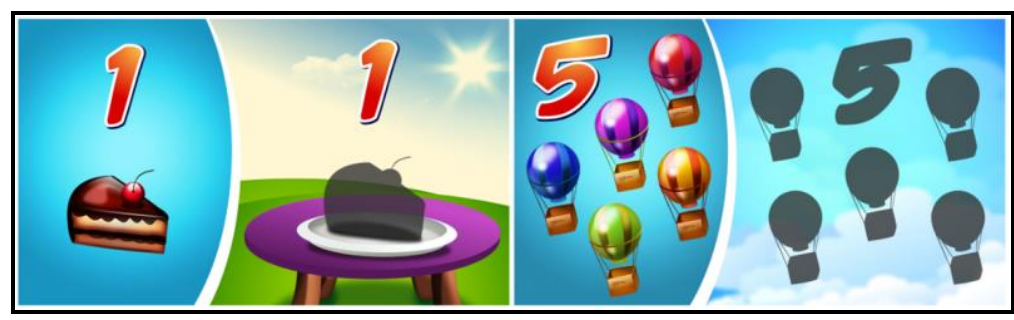

Figura 4. Atividades 1 e 5 do primeiro nível do aplicativo 123 Autismo.

Os próximos níveis apresentam dicas hierárquicas de cores que vão sendo retiradas conforme a progressão das ativididades do jogo. A cada fase superada, a dica visual torna menos evidente a solução esperada. Desta forma, é possível garantir que a criança aprenderá o conceito ao invés de apenas memorizar a possível solução. 
VIII Congresso Brasileiro de Informática na Educação (CBIE 2019)

Anais dos Workshops do VIII Congresso Brasileiro de Informática na Educação (WCBIE 2019)

No tocante às telas que compõem o Nível 2 do aplicativo, apresentadas na figura 5, o usuário será estimulado a associar a escrita dos números ao objeto correspondente, uma atividade que abrange basicamente atividades de letramento, partindo da área de armazenamento a área de execução, baseando-se nas dicas de cores. Quanto a área de armazenamento, os elementos atendem a recomendação do ensino estruturado e possuem posição aleatória a fim de evitar que a criança decore a ordem da possível execução da atividade.

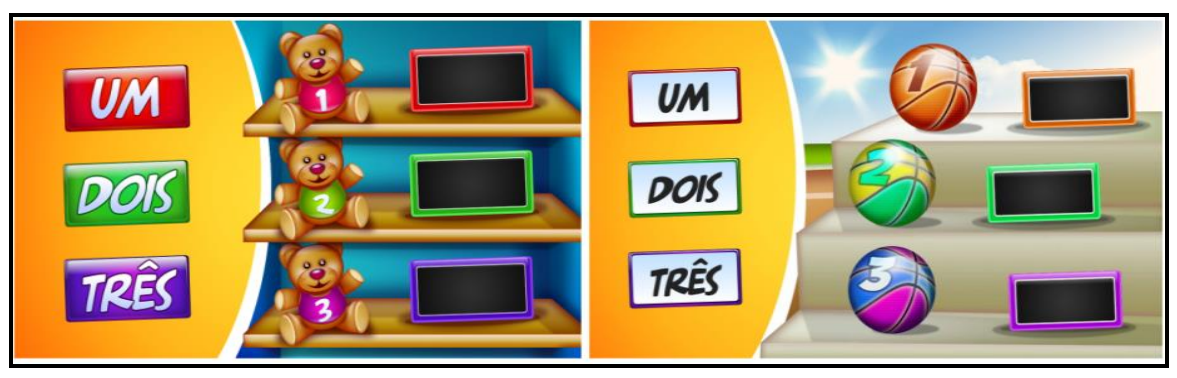

Figura 5. Atividades 1 e 5 do nível 2, com esvanecimento de dicas visuais.

Ainda se referindo ao Nível 2 e aos demais níveis que serão apresentados, o jogo atende a outra característica importante do Ensino Estruturado e valida os campos de resposta da atividade de forma que não permite que o usuário coloque um elemento na sua posição incorreta. Esta recomendação, denominada aprendizagem sem erro, visa auxiliar a criança com as dicas necessárias para que as suas ações obtenham sempre sucesso na execução da atividade. O Nível 3, por sua vez, segue a mesma estrutura dos níveis anteriores e possui a mesma base funcional, havendo apenas a mudança do objetivo. Neste nível, o usuário deve completar sequências numéricas, como as que são apresentadas na figura 6 .

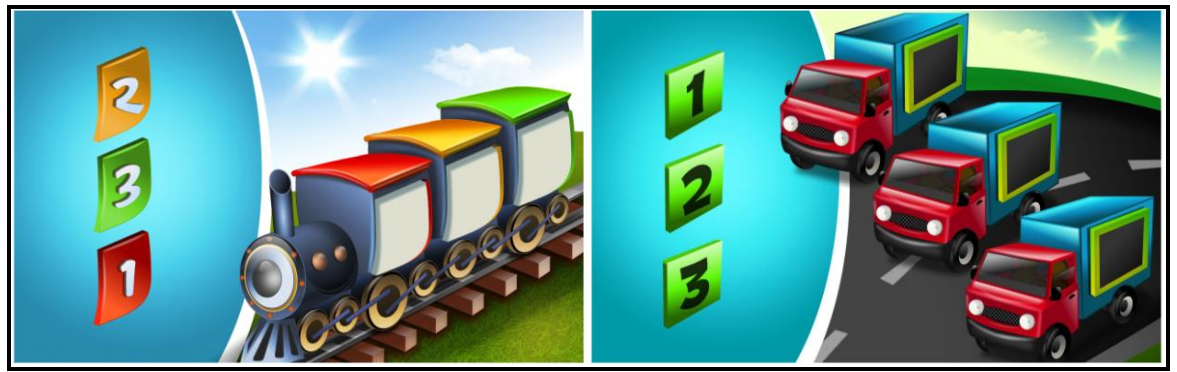

Figura 6. Atividades 1 e 5 do nível 3, com esvanecimento de dicas visuais.

As atividades do Nível 4 apresentam maior complexidade e abstração, onde o usuário precisa associar o número a sua quantidade e realizar somas, conforme apresentado na figura 7.

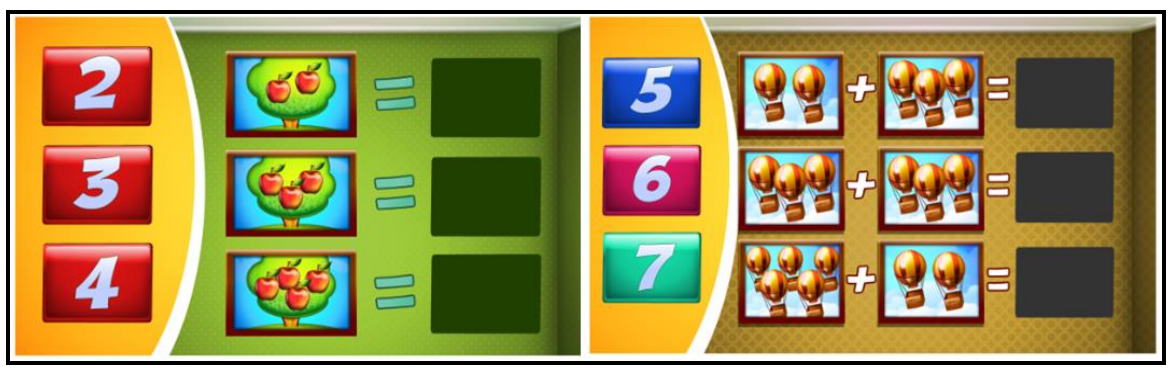

Figura 7. Atividades 1 e 5 do nível 4, com igualdade e soma. 
VIII Congresso Brasileiro de Informática na Educação (CBIE 2019)

Anais dos Workshops do VIII Congresso Brasileiro de Informática na Educação (WCBIE 2019)

Um vídeo demonstrativo do aplicativo pode ser visualizado através do YouTube, com o link: https://youtu.be/-dEWMRB xXQ. A instalação pode ser feita por meio do Google Play: https://play.google.com/store/apps/details?id=com.dokye.umdoistresautismo.

\section{Considerações finais}

A dinâmica de ensino-aprendizagem utilizada no jogo está devidamente representada através de níveis de complexidade ( 1 ao 4), visando auxiliar a criança autista a aprender de uma forma adaptada às suas necessidades e o seu grau de comprometimento, de acordo com as premissas do Ensino Estruturado. As vantagens da utilização do 123 Autismo são evidentes, visto que é oriundo de um processo sério de pesquisa realizado em parceria com profissionais capacitados com vasta experiência na área, o que de certa forma aumenta a credibilidade e confiabilidade na utilização do aplicativo dentro do processo interventivo e proporciona a transposição do ensino estruturado para os mais variados ambientes e sistemas operacionais, potencializando os efeitos da abordagem.

O 123 Autismo foi amplamente testado na fase de implementação, por oito crianças com autismo, de grau leve, moderado e severo, com níveis de aprendizado distintos, ao longo de 06 sessões, especialmente voltadas para a área de Pedagogia. Em cada sessão, com duração de 45 minutos, os indivíduos tiveram acesso ao tablet e foram observados aspectos subjetivos, como: um maior interesse e engajamento na execução das atividades propostas pelo jogo quando comparado ao engajamento pelas atividades tradicionais (em folha), atenção, concentração, comportamento e motivação de cada participante e também foram observados aspectos objetivos, como: tempo de execução, número de dicas necessárias, sejam elas verbais ou gestuais, e a quantidade de erros. Cada vez que utilizavam o aplicativo, as crianças demonstravam uma maior autonomia e a diminuição quantidade de erros devido ao entendimento da dinâmica do aplicativo, a eficiência da proposta e um maior aprendizado. Quando o jogo foi finalizado, com o design exclusivo e personalizado, o interesse e a atratividade das crianças se mantiveram. Os terapeutas da equipe multidisciplinar da associação parceira expressaram total concordância com os seguintes itens: beleza do design, adequabilidade da proposta ao público-alvo, aderência às premissas do ensino estruturado, atratividade das telas e facilidade de aprendizado e uso por parte das crianças com autismo.

Por fim, o 123 Autismo cumpre com o seu objetivo, que é oferecer uma proposta auxiliar para ensino de habilidades iniciais da matemática, através de interface agradável e ilustrações comuns ao seu dia a dia, bem como alinhamento com os programas terapêuticos utilizados para o público com autismo.

\section{Referências}

AMERICAN PSYCHIATRIC ASSOCIATION (APA). Manual diagnóstico e estatístico de transtornos mentais: DSM-5. Porto Alegre: Artmed, 2014. Disponível em: http://bit.ly/2Xz3prz. Acesso em 07/07/2019.

BELTRAME, W. A. R et al. Aventura Matemática. In: Congresso Brasileiro de Informática na Educação (CBIE), 7, 2018, pp 135-141, Fortaleza, Ceará. Anais... Fortaleza, 2018.

CORONA LABS. Corona: o mecanismo de jogo 2D, 2019. Página inicial. Disponível em: https://coronalabs.com. Acesso em 07/07/2019. 
VIII Congresso Brasileiro de Informática na Educação (CBIE 2019)

Anais dos Workshops do VIII Congresso Brasileiro de Informática na Educação (WCBIE 2019)

FARIAS, E. B.; SILVA, L. W.; CUNHA, M. X. C. Abc autismo: Um aplicativo móvel para auxiliar na alfabetização de crianças com autismo baseado no programa TEACCH. In: Simpósio Brasileiro de Sistemas de Informação, 10, pages 458-469, 2014, Londrina, Paraná. Anais... Londrina, 2014.

FERREIRA, M. M. M., FRANÇA, A. P. O Autismo e as Dificuldades no Processo de Aprendizagem Escolar. Revista Multidisciplinar e de Psicologia, V.11, N. 38. 2017.

GARCIA, P. M.; WELFER, D. ABC Fônico - Uma aplicação para auxiliar na alfabetização de crianças com TEA. In: Simpósio Brasileiro de Games (SBGames), 17, 2018, pp 1297-1300, Foz do Iguaçu, Paraná. Anais... Foz do Iguaçu, 2018.

GOBBO, M. R. M. et al. Jogo ACA para indivíduos com Transtorno do Espectro Autista. In: Simpósio Brasileiro de Games (SBGames), 17, 2018, pp 1114-1121, Foz do Iguaçu, Paraná. Anais... Foz do Iguaçu, 2018.

KWEE, C. S.; SAMPAIO, T. M. M. (2006) Abordagem Transdisciplinar no Autismo: O Programa TEACCH. Dissertação de Mestrado Profissionalizante em Fonoaudiologia. Universidade Veiga de Almeida. Rio de Janeiro - RJ.

MELlo, A. M et al. Retratos do Autismo. 1 ed. Secretaria Nacional da Pessoa com Deficiência, 2013. Disponível em http://bit.ly/2LP5Ofx. Acesso em 07/07/2019.

MESIBOV, G. B; SHEA, V. The TEACCH Program in the Era of Evidence-Based Practice. Journal of Autism and Developmental Disorders, May 2010, Volume 40, Issue 5, pp 570-579.

ORRÚ, S. E. Autismo, linguagem e educação: interação social no cotidiano escolar. Rio de Janeiro: Wak Ed., 2009.

PIRES, F. G. S. Tricô numérico: Um jogo para alfabetização matemática. In: Congresso Brasileiro de Informática na Educação (CBIE), 7, 2018, pp 249-255, Fortaleza, Ceará. Anais... Fortaleza, 2018.

REDERD, B. F.; SANTOS, R. P. L.; HEES, L. W. B. Autismo diante do raciocínio lógico matemático: Fatores determinantes e métodos de intervenção. Ensaios Pedagógicos (Sorocaba), vol.2, n.1, jan./abr. 2018, p.113-124.

SCHWARTZMAN, S. Transtornos do Espectro do Autismo (TEA). São Paulo: MEMNON, 2011.

SOCIEDADE BRASILEIRA DE PEDIATRIA. Manual de Orientação - Transtorno do Espectro do Autismo. Abril, 2019. Disponível em http://bit.ly/2Xyc6SE. Acesso em 07/07/2019. 